\title{
Ultrashort Pulsed Laser Machining for Biomolecule Trapping
}

\author{
Hae Woon Choi* \\ School of Mechanical and Automotive Engineering, Keimyung University, Daegu 704-701, Korea \\ Dave F. Farson \\ Laboratory for Multiscale Processing 8 Characterization, The Ohio State University, \\ Columbus 43210, OH, USA \\ L. James Lee \\ Department of Chemical and Biomolecular Engineering, The Ohio State University, \\ Columbus 43210, OH, USA \\ Ho Lee \\ School of Mechanical Engineering, Kyungpook National University, Daegu 702-701, Korea
}

(Received June 26, 2009 : revised September 10, 2009 : accepted September 14, 2009)

\begin{abstract}
Ultrashort pulse laser drilling of polycarbonate track-etched membrane ( $p$ TEM) material was used to fabricate a mouse embryo cell trapping device. Holes with a diameter of $2 \mu \mathrm{m}$ to $5 \mu \mathrm{m}$ were fabricated on a $10 \mu \mathrm{m}$ thick membrane using a femtosecond laser with a $150 \mathrm{fs}$ pulse width and $775 \mathrm{~nm}$ wavelength and multiple-pulse irradiation. In cell trapping tests, the overall cell occupancy of the machined holes in the fabricated $p$ TEM was found to be more than $80 \%$. The results of a single pulse and multiple pulse irradiation were compared in terms of the surface quality. It was generally found that a single pulse with high energy was less desirable than irradiation with multiple pulses of lower energy.
\end{abstract}

Keywords: Ultrashort laser, Femtosecond laser, Cell trapping, $p$ TEM

OCIS codes: (140.0140) Lasers and laser optics; (170.7160) Ultrafast technology

\section{INTRODUCTION}

Ultrashort pulse lasers have been widely used in biomedical device fabrication because their high peak intensity and non-thermal interaction allows high precision machining of many materials [1,2]. At this high intensity, the onset of optical breakdown that initiates ablation can be more deterministic than stochastic [3,4] and a wide range of materials can be machined $[5,6]$. For this reason, the ultrashort pulsed laser can offer advantages for dielectric material ablation or machining.

Compared to other lasers, there are many superiorities of ultrashort pulse lasers which have $\tau_{p}=150 \times 10^{-15}$ sec and $\lambda=775 \mathrm{~nm}$ of central wavelength for this experiment. The UV laser is one promising and fre-

*Corresponding author: hwchoi@kmu.ac.kr quently used laser for micromachining, but corrosive gas handling and UV radiation damages on the optics is still an issue, making it a less attractive tool for micro machining [5]. Use of a nanosecond laser with relatively longer pulse temporal duration was attempted for dielectric nano-fibers, however heat accumulation and irregular machining quality was found to be one challenging issue [16].

The cell transplantation or gene delivery has attracted many scientists and particularly biologists, due to its various applications in $\mathrm{BT}$ and NT technologies [3]. The gene delivery into a cell is one of the essential techniques in the cell therapy and many different approaches have been tried for the high production and secure delivery. Electroporation is an efficient method to introduce foreign impermeant molecules, such as drugs or genes suspended into a common medium, into 
cells. The conventional electroporation process is done with electroporators which create the high electric field through the cell solutions pipetted into a glass or plastic cuvettes with two electrodes [8-11]. Wang et al used micro-fluidic electroporation technology for delivery of small molecules and genes into cells [10]. While conventional electroporation has been based on the application of short electrical pulses (electropulsation), it is also possible to permeate live cells with a DC field [10].

While having advantages of versatility and efficiency, the electroporation process, as normally implemented, has a disadvantage of not being able to target specific cells for transfection. If specific cells are trapped from a solution for one-shot electroporation, the process can be more selective and useful. In this paper, we introduce a novel method to fabricate the cell trapping membranes using femtosecond laser drilling. It is demonstrated that ultrashort laser ablation can fabricate membranes useful for cell trapping. Electroporation plates with a holes a few micrometers in diameter were fabricated on $10 \mu \mathrm{m}$ thick track-etched membrane for mouse-embryo cell trapping. The effectiveness of cell trapping using the laser-machined membrane and the cell circulating chamber was tested.

\section{EXPERIMENT SETUP}

\section{Laser system setup}

The laser micromachining system comprised of a $\mathrm{Ti}: \mathrm{Al}_{2} \mathrm{O}_{3}$ femtosecond laser (Clark-MXR CPA2110) and a submicron resolution motion system (Parker Daedl MX80). The laser has a central wavelength of $775 \mathrm{~nm}$ with standard spectral bandwidth of $+/-25 \mathrm{~nm}$ and $3 \mathrm{kHz} / 6 \mathrm{kHz}$ switchable pulse repetition rates. The temporal pulse width $\left(\tau_{\mathrm{p}}\right)$ was $150 \mathrm{fs}$ (FWHM) and the average output power from the pulse compressor was measured to be $2.6 \mathrm{~W}$. The laser pulse has the horizontal polarization and the irradiance distribution of the laser output beam was nominally Gaussian with the beam diameter $\left(1 / \mathrm{e}^{2}\right)$ of $5 \mathrm{~mm}$. The quality factor $\left(\mathrm{M}^{2}\right)$ of the $\mathrm{x}$ axis and the $\mathrm{y}$ axis are 1.2 and 1.3, respectively. In order to adjust the output power from the laser, a series of optical attenuators was placed between the objective lens and the oscillator. The optical attenuators consist of two thin-film polarizing beam splitters (PBS) and a half wave plate. The laser pulse was gated externally by a high speed mechanical shutter with 4ms control time (NMlaser LS055).

The attenuated laser beam was focused on the target surface using a long working distance $(20.5 \mathrm{~mm})$ infinity corrected microscope objective lens with numerical aperture $\mathrm{NA}=0.42$. A coaxial vision system was integrated into the laser beam delivery optics to provide a capability for accurate focusing as well as in-situ monitoring. The focusing was adjusted by the

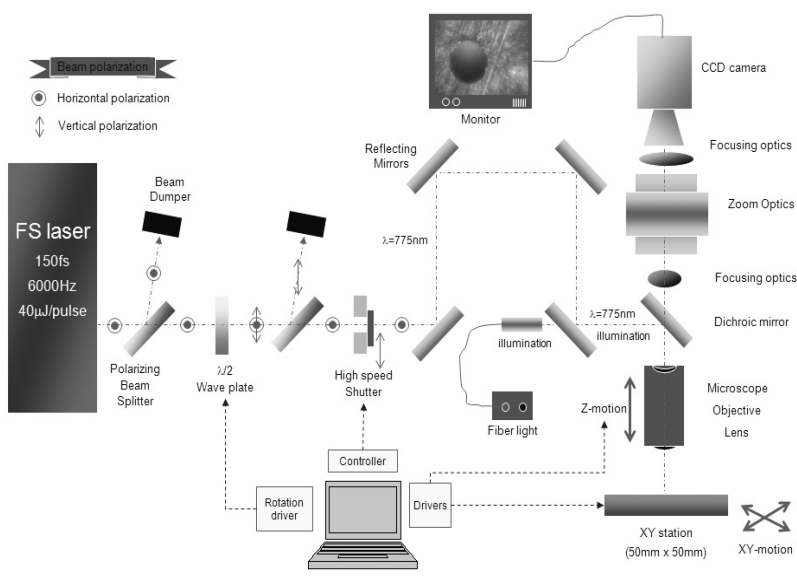

FIG. 1. Experiment setup for laser irradiation.

motorized $\mathrm{z}$ axis and a manual micrometer by visual inspection of a coaxial image generated by a CCD camera-based vision system. The motion system used in this experiment has the ultra high precision with 0.5 $\mu \mathrm{m}$ resolution with motion range of $50 \mathrm{~mm}$ for the $\mathrm{x}$ and y direction and $25 \mathrm{~mm}$ for the $\mathrm{z}$ direction. The axis was driven by linear motors controlled by a computer numerical control (CNC) system (Parker Compumotor ACR9000). The detail schematic of the experiment is shown in Fig. 1

\section{Scaffold fabrication and CNC code generation}

Polycarbonate track-etched membranes ( $p$ TEMs) with a thickness of $10 \mu \mathrm{m}$ were selected for fabrication of membranes for subsequent cell trapping studies. The $p$ TEM is very thin with tightly controlled pore size. It has been used for the micro-/nano scale filtration in many laboratory applications and recently widely used for the biomedical applications. As shown in Fig. 2(a), the $p$ TEM films have the intrinsic discrete pores that were produced during the manufacturing process by the combination of the charged particle bombardment, irradiation and chemical etching $[12,13]$. The distinct pore size of $p$ TEMs enables the capture of particles or cells, so its applications have been applied to biosensors, erythrocyte deformability testing, and other biomedical cell manipulations. The pore size of $p$ TEM is in the range of $100 \mathrm{~s}$ of $\mathrm{nm}$ which is quite suitable to capture the cells of about $2 \sim 5 \mu \mathrm{m}$ sized; however the pore locations are randomly distributed due to irregular ion particle distribution.

Since the $p$ TEM membrane is too thin to manipulate without support, the membrane was prepared on a plastic holder with a stretched shape as shown in Fig. 2(b). Because the focal depth of the objective lens used for laser machining was $1.6 \mu \mathrm{m}$, the plastic holder was not able to ensure the flatness of the $p$ TEM membrane necessary for consistent laser machining without focal point elevation adjusment. 


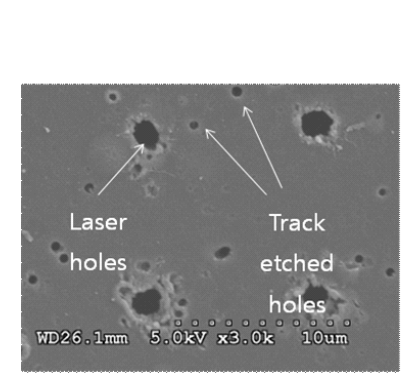

(a)

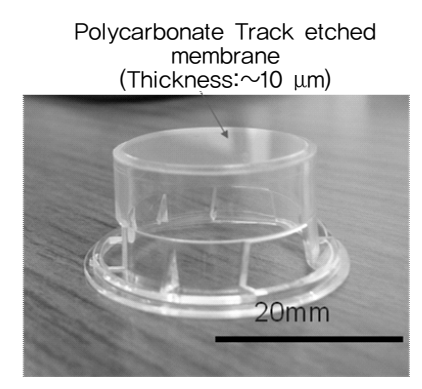

(b)
FIG. 2. Track-etched membranes on a plastic holder.

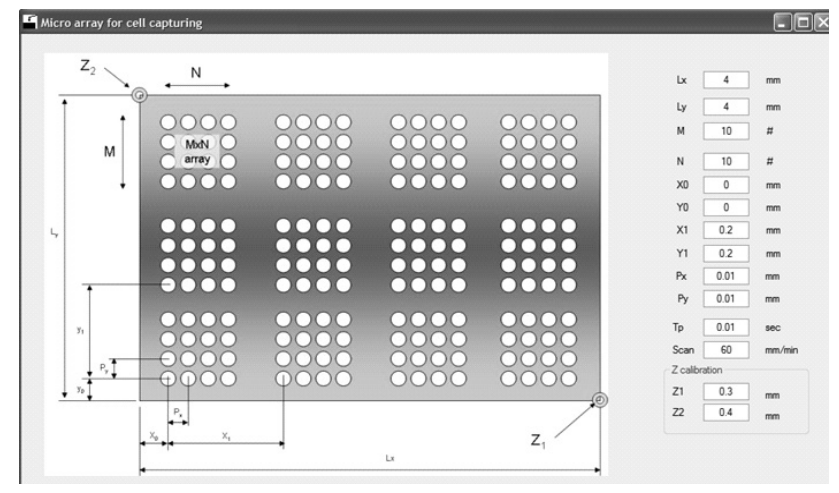

FIG. 3. GUI for microhole drilling program.

In order to implement the large-scale micromachining, the working distance between the focusing optic and $p$ TEMs needs to be kept constant, regardless of the membrane drilling locations. Since the surface of the stretched membrane was approximately flat, a simple linear expression shown in Eq. (1) was fit to the surface to allow compensation of the constant working distance.

$$
z=\frac{1}{c_{3}}\left(c_{1} x+c_{2} y+c_{4}\right)
$$

In Eq. (1), $c_{1}, c_{2}, c_{3}, c_{4}$ are constants and $\mathrm{x}, \mathrm{y}, \mathrm{z}$ are coordinates of drilling locations. With 3 known positions $(x, y, z)$, the three independent constants could be found from Eq. (1). The constants were entered in the motion control program so that the $\mathrm{z}$ locations could be automatically calculated with given $\mathrm{x}$ and $\mathrm{y}$ locations. A G-code program (CNC code) with fit parameters was generated for different $p$ TEMs samples and no out-of-focus drilling was observed across $20 \mathrm{~mm}$ diameter membrane surfaces. For convenience and flexibility in generating CNC programs based on a calibrated linear surface, a Visual Basic program coded with a GUI as shown in Fig. 3 was written.

The size of microholes can be also controlled by

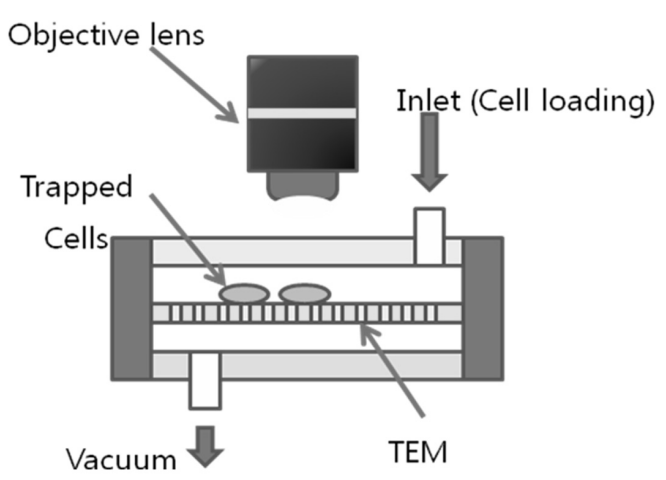

FIG. 4. Cell trapping experiment setup schematic.

changing the location of focusing. The objective lens which can maximize the focusing for a single wavelength with Numerical Aperture of 0.42 was selected for this experiment. Assuming the diffracting limited condition, the minimum spot diameter can be calculated as below [14],

$$
d=\frac{2 \lambda M^{2}}{\pi N A}
$$

where $d$ is focusing spot diameter, $N A$ is numerical aperture, $\lambda$ is wavelength of laser beam and $M^{2}$ is beam quality. With $M^{2}=1.3$, the diffraction limited spot diameter was estimated to be $1.5 \mu \mathrm{m}$.

\section{Cell trapping experiments}

A simple cell trapping device test bed was fabricated as shown in Fig. 4. the top and bottom sides are covered with clear PMMA which was machined by using a micro milling machine. The femtosecond laser machined $p$ TEMs with microholes were mounted in the middle of a trapping device. To inspect or monitor the cell trapping images, a microscope was located on the top of the device. While the backside of the membrane was kept in vacuum, live cells were loaded through the inlet of the device. Since the flatness of the membrane is very sensitive to the vacuum pressure, a manual syringe was used to apply vacuum pressure on the backside of the membrane.

\section{RESULTS}

\section{Single pulse irradiation (Single shot)}

The focus plane of the laser was set at the exact top location of the membrane and the single pulse of the femtosecond laser was applied with average power of $10 \mathrm{~mW}$. Since the pulse repetition rate of the laser was $3 \mathrm{kHz}$, the average pulse energy was $3.3 \mu \mathrm{J}$. The laser beam was scanned with velocity of $50 \mu \mathrm{m} / \mathrm{sec}$. Severe damage on the top surface of the membrane was 
observed, but no holes were drilled through to the bottom of the membrane. The single-pulse drilling test was repeated with the focus plane location adjusted to the bottom and the middle of the membrane. As in the top surface focusing case, severe damage on the surface was observed but no holes were drilled through the membrane.

\section{Multiple pulse irradiations}

In the next series of drilling tests, the membrane irradiated was multiple lower-energy laser pulses. The number of pulses passing through the mechanical shutter was measured with a photodiode. Due to the limitations of mechanical shutter response time $(\sim 10 \mathrm{~ms})$, a minimum of about 10 pulses could be gated onto the surface, however, the 10 pulse count as not quite accurate since some leading and tailing pulses were partially blocked during the shutter on/off transition. To minimize this effect, drilling tests were done with a minimum of 30 laser pulses.

In order to test the feasibility of microhole drilling with the multiple pulse irradiation, the membrane surface was irradiated with 30 pulses of various average powers. In case of the average power of $1 \mathrm{~mW}$ (energy of $0.33 \mu \mathrm{J}$ ), there was only slight damage to the top surface of the membrane. At $3 \mathrm{~mW}$ of laser power and above, holes were drilled completely through the membrane. The drilled membranes produced at various powers are arrayed in Figs. 5(a) - (d). As shown in Fig. 5(a) and (b), some burn patterns were observed by an optical microscope. Those burns were created when areas of the membrane were irradiated with weak laser

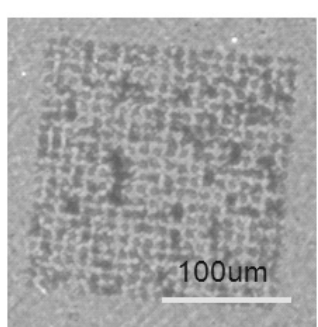

(a)

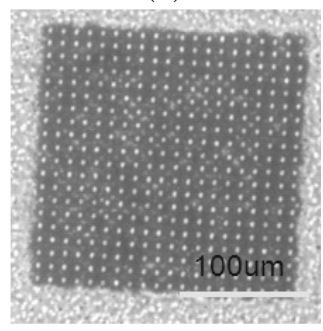

(c)

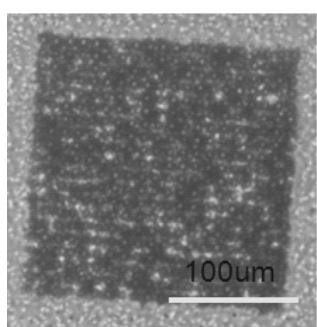

(b)

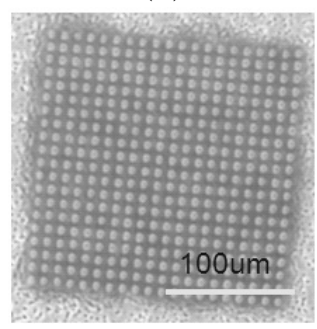

(d)
FIG. 5. Micro drilling results with Optical Microscope, 30 shots (a) $\mathrm{P}_{\text {avg }}=1 \mathrm{~mW}$ (b) $\mathrm{P}_{\text {arg }}=2 \mathrm{~mW}$ (c) $\mathrm{P}_{\text {avg }}=3 \mathrm{~mW}$ (d) $\mathrm{P}_{\text {avg }}=4 \mathrm{~mW}$.

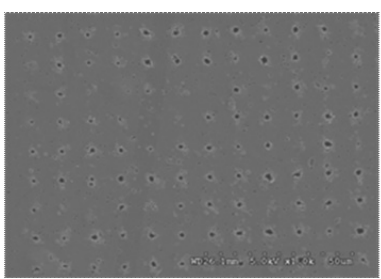

(a)

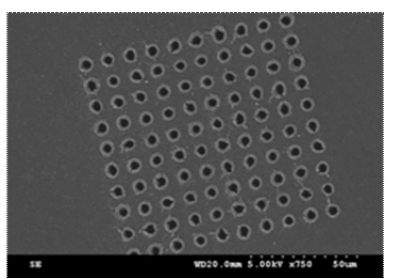

(b)
FIG. 6. SEM images of laser drilled holes (a) $\mathrm{P}_{\text {avg }}=2$ $\mathrm{mW}$ (b) $\mathrm{P}_{\text {avg }}=3 \mathrm{~mW}$.

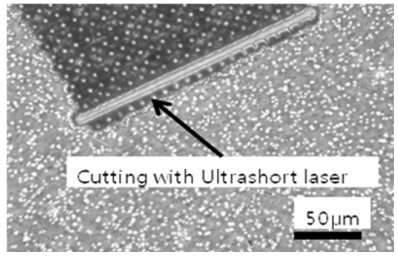

$-(\mathrm{a})$

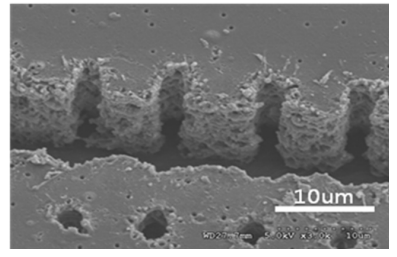

(b)
FIG. 7. Cross-section of drilled holes.

power. The energy intensity was not high enough to ablate the material but changed optical properties to produce more opaqueness.

To characterize the quality of drilled holes, scanning electron microscope (SEM) images were taken for both $2 \mathrm{~mW}$ and $3 \mathrm{~mW}$ power as shown in Fig. 6. Complete holes were fabricated with $2 \mathrm{~mW}$ of laser power although their diameter was somewhat variable. Laser power of $3 \mathrm{~mW}$ produced larger holes with more consistent diameter. The $2 \mathrm{~mW}$ laser power was used in subsequent tests because there was less melted material surrounding the holes.

\section{Thoroughness of the hole in the microscopy image}

To assess the completeness and consistence of drilled holes, some samples were cut using ultrashort laser scanning as shown in Fig. 7(a). The cross sections of drilled holes were characterized using SEM images as shown in Fig. 7(b). As seen in the figure, the diameters of the drilled holes were relatively uniform and the holes passed completely through the thickness.

\section{Cell trapping experiments}

The effectiveness of the fabricated $p$ TEMs for cell capturing was tested with mouse embryonic stem cells. Vacuum was applied to the backside of the membrane and the front side was coated with the cells in solution (Fig. 8a). Live images of cell trapping were captured with an optical microscope as shown in Fig. 8(b). The percentage of the holes in the array occupied by cells was calculated to be as high as $80 \%$. The fraction of occupied holes is expected to increase with higher concentration of cells in the solutions. 


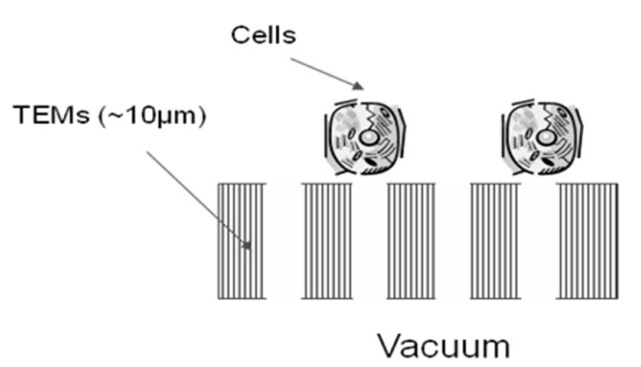

(a)

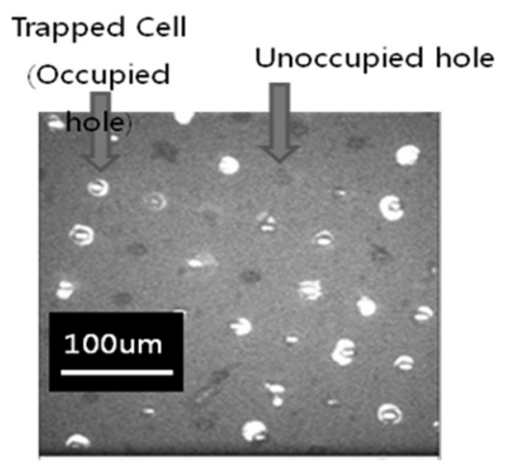

(b)

FIG. 8. (a) Cell capturing principle (b) trapped cells on $p$ TEM.

\section{CONCLUSION}

Femtosecond laser drilling was effectively used to fabricate track-etched membranes for cell trapping. Holes with $2 \mu \mathrm{m}$ to $5 \mu \mathrm{m}$ diameter on $10 \mu \mathrm{m}$ thick track-etched membrane and the minimum required power were suggested to be $2 \mathrm{~mW}$. The occupancy of the trapped cells in the holes was over $80 \%$. The drilled microholes were evaluated and turned out to be very straight along with the depth. A single shot with high power was less desirable than low power with multi shots. A simple plane equation was introduced to compensate the working distance between the objective lens and the membrane surface which was effective fordrilling the holes on focus locations.

\section{DISCUSSION}

Transparent and dielectric membrane drilling has been a challenging research task and was effectively solved by the femtosecond laser. The $p$ TEM is a breathable film which has arbitrary nanoscale holes, but we used it for a cell trapping membrane with regular holes by a femtosecond laser process. With its ultrahigh energy density, the femtosecond laser directly machined a hole on the designated spot without damaging neighboring materials. The aspect ratio of the micro-holes was measured to be up to $5(2 \mu \mathrm{m}$ diameter in $10 \mu \mathrm{m}$ thick film) and the size was controllable by adjusting laser power. Some marginal burn patterns were found around the drilled holes due to ablation and damaging threshold, and further research needs to be planned to minimize the damaged area. The developed membrane can be packaged and used as a biosensor for gene-therapy as well as cell trapping. Coating the surface area with SAM (Self Assembled Mono-layer) may provide more functional device for biomedical device applications.

\section{ACKNOWLEDGEMENT}

This research was supported by the National Science Foundation under Grant No. EEC-0425626, new faculty research awards by Korea Research Foundation (KRF2008-331-D00097), the Korea Science and Engineering Foundation (KOSEF) grants funded by the Korea government (MEST) (R01-2007-000-11427-0) and by the Korean Ministry of Education, Science and Technology (The Regional Core Research Program/Medical Convergence Technology Develop-ment Consortium for Anti-aging and Well-being)

\section{REFERENCES}

1. B. Stuart, M. Feit, A. Rubenchik, B. Shore, and M. Perry, "Laser-induced damage in dielectrics with nanosecond to subpicosecond pulses," Physical Review Letters 74, 2248-2252 (1995).

2. S. Nolte, C. Momma, H. Jacobs, A. Tunnermann, B. Chichkov, B. Wellegehausen, and H. Welling, "Ablation of metals by ultrashort laser pulses,” J. Opt. Soc. Am. B 14, 2716-2722 (1997).

3. J. Acker, "Bio-preservation of cells and engineered tissues," in Tissue Engineering II, T. Scheper, K. Lee, and D. Kaplan, ed. (Springer, Berlin, Germany, 2005).

4. A. Garner, G. Chen, N. Chen, V. Sridhara, J. Kolb, R. Swanson, S. Beebe, R. Joshi, and K. Schoenbach, "Ultrashort electric pulse induced changes in cellular dielectric properties," Biochem. Biophy. Research Comm. 362, 139-144 (2007).

5. X. Liu, D. Du, and G. Mourou, "Laser ablation and micromachining with ultrashort laser pulses, " IEEE J. Quantum Electron. 33, 1706-1716 (1997).

6. D. Du, X. Liu, G. Korn, J. Squier, and G. Mourou, "Laser induced breakdown by impact ionization in $\mathrm{SiO}_{2}$ with pulse widths from 7 ns to $150 \mathrm{fs}$," Appl. Phys. Lett. 64, 3071-3073 (1994).

7. V. Kohli, J. Acker, and A. Elezzabi, "Reversible permeabilization using high-intensity femtosecond laser pulses: applications to biopreservation,” Biotechnol. Bioeng. 92, 889-899 (2005).

8. L. MacQueen, M. Bushmann, and M. Wertheimer, "Gene delivery by electroporation after dielectro-phoretic 
positioning of cells in a non-uniform electric field," Bioelectrochemistry 72, 141-148 (2008).

9. J. Zhang, P. Blackmore, B. Hargrave, S. Xiao, S. Beebe, and K. Schoenbach, "Nanosecond pulse electric field: a novel non-ligand agonist for platelet activation," Archives of Biochemistry and Biophysics 471, 240-248 (2008).

10. H. Wang and C. Lu, "Microfluidic electroporation for delivery of small molecules and genes into cells using a common DC power supply," Biotechnol. Bioeng. 100, 579-586 (2008).

11. Wikipedia, http://en.wikipedia.org/wiki/Electroporation

12. http://www.whatman.com/
13. http://www.2spi.com/catalog/spec prep/filter3.html

14. M. Sasnett, "Propagation of multimode laser beams the M2 factor," in The Physics and Technology of Laser Resonators, D. Hall and P. Jackson, ed. (IOP Publishing, Bristol, UK, 1989), pp. 132-142.

15. K. Salonitis, A. Stournaras, G. Tsoukantas, P. Stavropoulos, and G. Chryssolouris, "A theoretical and experimental investigation on limitations of pulsed laser drilling," J. Mat. Proc. Technology 183, 96-103 (2007).

16. H. Choi, J. Johnson, J. Nam, D. Farson, and J. Lannutti, "Structuring electrospun polycaprolactone nanofiber tissue scaffolds by femtosecond laser ablation," J. Laser Applications 19, 225-231 (2007). 